\title{
TRANSFORMS ON WHITE NOISE FUNCTIONALS WITH THEIR APPLICATIONS TO CAUCHY PROBLEMS
}

\author{
DONG MYUNG CHUNG ${ }^{1}$ AND UN CIG JI
}

\begin{abstract}
A generalized Laplacian $\Delta_{G}(K)$ is defined as a continuous linear operator acting on the space of test white noise functionals. Operator-parameter $\mathcal{G}_{A, B^{-}}$and $\mathcal{F}_{A, B}$-transforms on white noise functionals are introduced and then prove a characterization theorem for $\mathcal{G}_{A, B}$ and $\mathcal{F}_{A, B}$-transforms in terms of the coordinate differential operator and the coordinate multiplication. As an application, we investigate the existence and uniqueness of solution of the Cauchy problem for the heat equation associated with $\Delta_{G}(K)$.
\end{abstract}

\section{$\S 1$. Introduction}

Gross [5] initiated the study of the theory of differential equations on infinite dimensional abstract Wiener space $(H, B)$. Suppose $\varphi$ is a twice $H$-differentiable function on $B$ such that its second $H$-derivative $\varphi^{\prime \prime}$ is a trace class operator of $H$. Then the Gross Laplacian $\Delta_{G} \varphi$ of $\varphi$ is defined by

$$
\Delta_{G} \varphi=\operatorname{trace}_{H} \varphi^{\prime \prime}
$$

In [5] Gross studied the solution of the Cauchy problem for the heat equation associated with the Gross Laplacian $\Delta_{G}$ :

$$
\frac{\partial u(x, \theta)}{\partial \theta}=\frac{1}{2} \Delta_{G} u(x, \theta) .
$$

It has been shown [5] that the solution can be represented as an integral with respect to the abstract Wiener measure. For further works see [10], [15], [19].

Based on the white noise analysis, Kuo [11] formulated the Gross Laplacian $\Delta_{G}$ and the number operator $N$ in terms of the Hida differentiation $\partial_{t}$

Received December 11, 1995.

${ }^{1}$ Research supported by KOSEF 951-0102-001-2 and BSRIP 96-1412. 
and its adjoint $\partial_{t}^{*}$. In [1] Chung and Ji obtained an explicit description of differentiable one-parameter transformation group whose infinitesimal generator is $a \Delta_{G}+b N$ and applied this result to study Cauchy problem for the heat equation associated with $a \Delta_{G}+b N$ on white noise space.

In this paper we first introduce a generalized Laplacian $\Delta_{G}(K)$, called the $K$-Gross Laplacian, which is a continuous linear operator acting on the space $(E)$ of test white noise functionals and next introduce operatorparameter $\mathcal{G}_{A, B^{-}}$and $\mathcal{F}_{A, B^{-}}$-transforms on white noise functionals as a generalization of scalar-parameter $\mathcal{G}_{\alpha, \beta^{-}}$and $\mathcal{F}_{\alpha, \beta^{-}}$transforms studied in $[1,2]$. The purpose of this paper is to extend some results in [1] for the $\mathcal{G}_{A, B^{-}}$and $\mathcal{F}_{A, B}$-transforms and to investigate the solution of the Cauchy problem for the heat equation associated with the $K$-Gross Laplacian $\Delta_{G}(K)$.

The paper is organized as follows. In Section 2, we give a brief background and known results in white noise analysis. In Section 3, we define the $K$-Gross Laplacian $\Delta_{G}(K)$ and study the properties of $K$-Gross Laplacian. In Section 4, using the characterization theorem for the symbol of an operator acting on the space $(E)$ due to Obata [18], we define an operatorparameter transform $\mathcal{G}_{A, B}$ acting on $(E)$ and then define the $\mathcal{F}_{A, B}$-transform as $\mathcal{F}_{A, B}=\mathcal{G}_{A, B}^{*}$. In Section 5 , we prove a characterization theorem for $\mathcal{G}_{A, B}$ and $\mathcal{F}_{A, B}$-transforms in terms of the coordinate differential operator and the coordinate multiplication. In Section 6 , we explicitly obtain a differentiable one-parameter transformation group with infinitesimal generator $\Delta_{G}(K)$ and use this result to study the existence and uniqueness of the solutions of Cauchy problems for the heat equation associated with the generalized Gross Laplacian $\Delta_{G}(K)$.

\section{§. Preliminaries}

In this section we shall briefly recall some of concepts, notation and known results in white noise analysis $[1,2,8,14,18]$. Let $T$ be a topological space with a Borel measure $\nu(d t)=d t$. Let $H=L^{2}(T, \nu)$ be the real Hilbert space of all square integrable functions on $T$ with norm $|\cdot|_{0}$. Let $A$ be a densely defined self-adjoint operator in $H$. We assume that there is an orthonormal basis $\left\{e_{j}\right\}_{j=0}^{\infty}$ for $H$ contained in the domain of $A$ such that

$$
A e_{j}=\lambda_{j} e_{j}, \quad j=0,1, \ldots,
$$




$$
1<\lambda_{0} \leq \lambda_{1} \leq \cdots \rightarrow \infty, \text { and } \sum_{j=0}^{\infty} \lambda_{\jmath}^{-2}<\infty .
$$

In the sequel the following two constants will be used:

$$
\rho=\left\|A^{-1}\right\|_{\mathrm{OP}}=\lambda_{0}^{-1}, \quad \delta=\left\|A^{-1}\right\|_{\mathrm{HS}}=\left(\sum_{j=0}^{\infty} \lambda_{\jmath}^{-2}\right)^{1 / 2} .
$$

Note that $0<\rho<1, \rho<\delta$. For each $p \geq 0$, define

$$
|\xi|_{p}^{2}=\left|A^{p} \xi\right|_{0}^{2}=\sum_{j=0}^{\infty} \lambda_{j}^{2 p}\left\langle\xi, e_{j}\right\rangle^{2}
$$

and let $E_{p}=\left\{\xi \in H:|\xi|_{p}<\infty\right\}$. Then $E_{p}$ is a real separable Hilbert space with the norm $|\cdot|_{p}$. It is easily seen that $E_{p} \subset E_{q}$ for any $p \geq q \geq 0$ and the inclusion map $E_{p+1} \hookrightarrow E_{p}$ is of Hilbert-Schmidt type for any $p \geq 0$. Let $E$ be the projective limit of $\left\{E_{p}: p \geq 0\right\}$ and $E^{*}$ the topological dual space of $E$. Then $E$ becomes a nuclear space since $0<\delta<\infty$ by assumption, and we get a Gel'fand triple $E \subset H \subset E^{*}$ with the continuous inclusions:

$$
E \subset E_{p} \subset H \subset E_{p}^{*} \subset E^{*}, \quad p \geq 0 .
$$

It is known that $E^{*}$ is the inductive limit of $\left\{E_{p}^{*}: p \geq 0\right\}$ and that the inductive limit topology of $E^{*}$ coincides with the strong dual topology. We denote by $\langle\cdot, \cdot\rangle$ the canonical bilinear form on $E^{*} \times E$.

We further assume that the Gel'fand triple $E \subset H \subset E^{*}$ satisfies the hypotheses (H1)-(H3):

(H1) For each $\xi \in E$ there exists a unique continuous function $\tilde{\xi}$ on $T$ such that $\xi(t)=\widetilde{\xi}(t)$ for $\nu$-a.e. $t \in T$.

(H2) For each $t \in T$ the evaluation map $\delta_{t}: \xi \rightarrow \xi(t), \xi \in E$, is a continuous linear functional, i.e., $\delta_{t} \in E^{*}$.

(H3) The map $t \rightarrow \delta_{t} \in E^{*}, t \in T$, is continuous with respect to the strong dual topology of $E^{*}$.

Let $\mu$ be the Gaussian measure on $E^{*}$ whose characteristic functional is given by

$$
\int_{E^{*}} \exp \{i\langle x, \xi\rangle\} \mu(d x)=\exp \left\{-\frac{1}{2}|\xi|_{0}^{2}\right\}, \quad \xi \in E .
$$


We denote by $\left(L^{2}\right) \equiv L_{\mathbb{C}}^{2}\left(E^{*}, \mu\right)$ the complex Hilbert space of $\mu$-square integrable functions on $E^{*}$ with norm $\|\cdot\|_{0}$. By the Wiener-Itô decomposition theorem, each $\phi \in\left(L^{2}\right)$ admits an expression

$$
\phi(x)=\sum_{n=0}^{\infty}\left\langle: x^{\otimes n}:, f_{n}\right\rangle, \quad x \in E^{*},
$$

where $f_{n} \in H_{\mathbb{C}}^{\widehat{\otimes} n}$ (the $n$-fold symmetric tensor product of the complexification of $H$ ) and $: x^{\otimes n}$ : denotes the Wick ordering of $x^{\otimes n}$ (see [8]). Moreover, the $\left(L^{2}\right)$-norm $\|\phi\|_{0}$ of $\phi$ is given by

$$
\|\phi\|_{0}=\left(\sum_{n=0}^{\infty} n !\left|f_{n}\right|_{0}^{2}\right)^{\frac{1}{2}}
$$

where $|\cdot|_{0}$ denotes the $H_{\mathbb{C}}^{\otimes n}$-norm for any $n$.

Let $\Gamma(A)$ denote the second quantization operator of $A$ which is densely defined on $\left(L^{2}\right)$ as follows: For $\phi(x)=\sum_{n=0}^{\infty}\left\langle: x^{\otimes n}:, f_{n}\right\rangle, \Gamma(A) \phi(x)$ is given by

$$
\Gamma(A) \phi(x)=\sum_{n=0}^{\infty}\left\langle: x^{\otimes n}:, A^{\otimes n} f_{n}\right\rangle .
$$

For each $p \in \mathbb{R}$, define

$$
\|\phi\|_{p}=\left\|\Gamma(A)^{p} \phi\right\|_{0}=\left(\sum_{n=0}^{\infty} n !\left|f_{n}\right|_{p}^{2}\right)^{\frac{1}{2}} .
$$

Let $\left(E_{p}\right)=\left\{\phi \in\left(L^{2}\right):\|\phi\|_{p}<\infty\right\}$. Then $\left(E_{p}\right)$ is a Hilbert space with the norm $\|\cdot\|_{p}$. Obviously, $\left(E_{p}\right) \subset\left(E_{q}\right)$ for any $p \geq q \geq 0$. Let $(E)$ be the projective limit of $\left\{\left(E_{p}\right): p \geq 0\right\}$ and $(E)^{*}$ the topological dual space of $(E)$. Then $(E)$ is a nuclear space and we have a Gel'fand triple $(E) \subset\left(L^{2}\right) \subset(E)^{*}$ with the following continuous inclusions:

$$
(E) \subset\left(E_{p}\right) \subset\left(L^{2}\right) \subset\left(E_{p}\right)^{*} \subset(E)^{*}, \quad p \geq 0 .
$$

It is known that $(E)^{*}$ is the inductive limit of $\left\{\left(E_{p}\right)^{*}: p \geq 0\right\}$ and that the inductive topology of $(E)^{*}$ coincides with the strong dual topology. An element in $(E)$ (resp. in $\left.(E)^{*}\right)$ is called a test (resp. generalized) white noise functional. It is obvious that $\phi \in\left(L^{2}\right)$ belongs to $(E)$ if and only if $f_{n} \in E_{\mathbb{C}}^{\hat{\otimes} n}$ 
for all $n$ and $\|\phi\|_{p}=\left(\sum_{n=0}^{\infty} n !\left|f_{n}\right|_{p}^{2}\right)^{1 / 2}<\infty$ for all $p \geq 0$. We denote by $\langle\langle\cdot, \cdot\rangle\rangle$ the canonical $\mathbb{C}$-bilinear form on $(E)^{*} \times(E)$. For each $\Phi \in(E)^{*}$, there exists a unique sequence $\left\{F_{n}\right\}_{n=0}^{\infty}, F_{n} \in\left(E_{\mathbb{C}}^{\otimes n}\right)_{\mathrm{sym}}^{*}$ such that

$$
\langle\langle\Phi, \phi\rangle\rangle=\sum_{n=0}^{\infty} n !\left\langle F_{n}, f_{n}\right\rangle
$$

for all $\phi \in(E)$ with $\phi(x)=\sum_{n=0}^{\infty}\left\langle: x^{\otimes n}:, f_{n}\right\rangle, f_{n} \in E_{\mathbb{C}}^{\widehat{\otimes} n}$. Moreover $\sum_{n=0}^{\infty} n$ ! $\left|F_{n}\right|_{-p}^{2}<\infty$ for some $p \geq 0$. In this case we use a formal expression for $\Phi \in(E)^{*}:$

$$
\Phi(x)=\sum_{n=0}^{\infty}\left\langle: x^{\otimes n}:, F_{n}\right\rangle, \quad x \in E^{*} .
$$

For each $\xi \in E_{\mathbb{C}}$, an exponential vector $\phi_{\xi}$ is defined by

$$
\phi_{\xi}(x)=\sum_{n=0}^{\infty}\left\langle: x^{\otimes n}:, \frac{\xi^{\otimes n}}{n !}\right\rangle, \quad x \in E^{*} .
$$

It is well-known that $\left\{\phi_{\xi}: \xi \in E_{\mathbb{C}}\right\}$ spans a dense subspace of $(E)$.

The $S$-transform of $\Phi \in(E)^{*}$ is a function on $E_{\mathbb{C}}$ defined by

$$
S \Phi(\xi)=\left\langle\left\langle\Phi, \phi_{\xi}\right\rangle, \quad \xi \in E_{\mathbb{C}} .\right.
$$

For $F_{m} \in\left(E_{\mathbb{C}}^{\otimes m}\right)^{*}$ and $f_{m+n} \in E_{\mathbb{C}}^{\widehat{\otimes}(m+n)}$, we denote by $F_{m} \otimes_{m} f_{m+n} \in$ $E_{\mathbb{C}}^{\widehat{\otimes} n}$ uniquely determined by

$$
\left\langle G_{n} \otimes F_{m}, f_{m+n}\right\rangle=\left\langle G_{n}, F_{m} \otimes_{m} f_{m+n}\right\rangle, \quad G_{n} \in\left(E_{\mathbb{C}}^{\otimes n}\right)^{*}
$$

where $F_{m} \otimes_{m} f_{m+n}$ is a left contraction (see [18]).

Let $\phi \in(E)$ be given by $\phi(x)=\sum_{n=0}^{\infty}\left\langle: x^{\otimes n}:, f_{n}\right\rangle$. For $y \in E^{*}$, put

$$
D_{y} \phi(x)=\lim _{\theta \rightarrow 0} \frac{\phi(x+\theta y)-\phi(x)}{\theta}, \quad x \in E^{*} .
$$

It is known that the limit always exists and $D_{y} \phi$ is given by

$$
D_{y} \phi(x)=\sum_{n=0}^{\infty} n\left\langle: x^{\otimes n-1}:, y \widehat{\otimes}_{1} f_{n}\right\rangle, \quad x \in E^{*} .
$$

Furthermore, $D_{y}$ becomes a continuous linear operator from $(E)$ into itself. This operator $D_{y}$ is called a Gâteaux differential operator in the direction 
$y \in E^{*}$. Since the delta functions $\delta_{t}$ are in $E^{*}$ by $(\mathrm{H} 2)$, we define $\partial_{t}=D_{\delta_{t}}$, $t \in T$. This operator $\partial_{t}$ is called Hida's differential operator.

Let $q_{\eta}, \eta \in E$ denote the multiplication by $\langle\cdot, \eta\rangle$. Then $q_{\eta}$ is a continuous linear operator from $(E)$ into itself. Moreover, we have $q_{\eta}=D_{\eta}+D_{\eta}^{*}$.

Let $L\left((E),(E)^{*}\right)$ (resp. $L((E),(E))$ ) denote the space of all continuous linear operator from $(E)$ into $(E)^{*}$ (resp. from $(E)$ into itself). For $\phi$, $\psi \in(E)$, define a function by

$$
h_{\phi, \psi}\left(s_{1}, \cdots, s_{l}, t_{1}, \cdots, t_{m}\right)=\left\langle\left\langle\partial_{s_{1}}^{*} \cdots \partial_{s_{l}}^{*} \partial_{t_{1}} \cdots \partial_{t_{m}} \phi, \psi\right\rangle\right\rangle .
$$

Then $h_{\phi, \psi} \in E_{\mathbb{C}}^{\otimes(l+m)}$, and for any $\kappa \in\left(E_{\mathbb{C}}^{\otimes(l+m)}\right)^{*}$, there exists a continuous linear operator $\Xi_{l, m}(\kappa) \in L\left((E),(E)^{*}\right)$ such that

$$
\left\langle\left\langle\Xi_{l, m}(\kappa) \phi, \psi\right\rangle\right\rangle=\left\langle\kappa, h_{\phi, \psi}\right\rangle, \quad \phi, \psi \in(E) .
$$

We sometimes use a formal integral expression:

$$
\begin{aligned}
& \Xi_{l, m}(\kappa) \\
& \quad=\int_{T^{l+m}} \kappa\left(s_{1}, \cdots, s_{l}, t_{1}, \cdots, t_{m}\right) \partial_{s_{1}}^{*} \cdots \partial_{s_{l}}^{*} \partial_{t_{1}} \cdots \partial_{t_{m}} d s_{1} \cdots d s_{l} d t_{1} \cdots d t_{m} .
\end{aligned}
$$

We call $\Xi_{l, m}(\kappa)$ an integral kernel operator with kernel distribution $\kappa$ (see $[8,18])$.

For any $\Xi \in L\left((E),(E)^{*}\right)$, a function $\widehat{\Xi}$ on $E_{\mathbb{C}} \times E_{\mathbb{C}}$ defined by

$$
\widehat{\Xi}(\xi, \eta)=\left\langle\left\langle\Xi \phi_{\xi}, \phi_{\eta}\right\rangle\right\rangle, \quad \xi, \eta \in E_{\mathbb{C}}
$$

is called the symbol of $\Xi$. We note that the map $\Xi \rightarrow \widehat{\Xi}$ is injective.

The following theorem is an analytic characterization theorem for symbols of operators on white noise functionals due to Obata [18].

Proposition 2.1. Let $\Theta$ be a function on $E_{\mathbb{C}} \times E_{\mathbb{C}}$ with values in $\mathbb{C}$. Then there exists a continuous operator $\Xi \in L((E),(E))$ such that $\Theta=\widehat{\Xi}$ if and only if

(i) for any $\xi, \xi_{1}, \eta, \eta_{1} \in E_{\mathbb{C}}$, the function

$$
z, w \longrightarrow \Theta\left(z \xi+\xi_{1}, w \eta+\eta_{1}\right)
$$

is an entire holomorphic function on $\mathbb{C} \times \mathbb{C}$; 
(ii) for any $p \geq 0$ and $\epsilon>0$, there exist constants $C \geq 0$ and $q \geq 0$ such that

$$
|\Theta(\xi, \eta)| \leq C \exp \left\{\epsilon\left(|\xi|_{p+q}^{2}+|\eta|_{-p}^{2}\right)\right\}, \quad \xi, \eta \in E_{\mathbb{C}}
$$

In this case,

$$
\|\Xi \phi\|_{p-1} \leq C M(\epsilon, q, r)\|\phi\|_{p+q+r+1}, \quad \phi \in(E),
$$

where $M(\epsilon, q, r)$ is a (finite) constant for $\epsilon<\left(2 e^{3} \delta^{2}\right)^{-1}, r \geq r_{0}(q) \geq 0$.

Proposition 2.2. ([18]) For any $\Xi \in L\left((E),(E)^{*}\right)$, there exists a unique family of distributions $\left\{\kappa_{l, m}\right\}_{l, m=0}, \kappa_{l, m} \in\left(E_{\mathbb{C}}^{\otimes(l+m)}\right)_{\mathrm{sym}(l, m)}^{*}$ such that

$$
\Xi=\sum_{l, m=0}^{\infty} \Xi_{l, m}\left(\kappa_{l, m}\right),
$$

where the series converges in $L\left((E),(E)^{*}\right)$. If $\Xi \in L((E),(E))$, then $\kappa_{l, m} \in$ $\left(E_{\mathbb{C}}^{\widehat{\otimes} l}\right) \otimes\left(E_{\mathbb{C}}^{\otimes m}\right)_{\text {sym }}^{*}$ and the series $(2.1)$ converges in $L((E),(E))$.

The unique expression given in (2.1) is referred to as the Fock expansion of $\Xi$.

\section{$\S 3$. Generalized Gross Laplacians}

Let $L\left(E_{\mathbb{C}}, E_{\mathbb{C}}^{*}\right)$ and $L\left(E_{\mathbb{C}}, E_{\mathbb{C}}\right)$ be the set of all continuous linear operators from $E_{\mathbb{C}}$ into $E_{\mathbb{C}}^{*}$ and those from $E_{\mathbb{C}}$ into itself, respectively. In this section, we define, for $K \in L\left(E_{\mathbb{C}}, E_{\mathbb{C}}^{*}\right)$, a generalized Gross Laplacian $\Delta_{G}(K)$, called the $K$-Gross Laplacian.

We denote by $\tau(K)$ the corresponding distribution to $K \in L\left(E_{\mathbb{C}}, E_{\mathbb{C}}^{*}\right)$ under the canonical isomorphism $L\left(E_{\mathbb{C}}, E_{\mathbb{C}}^{*}\right) \cong\left(E_{\mathbb{C}} \otimes E_{\mathbb{C}}\right)^{*}$, i.e.

$$
\langle\tau(K), \xi \otimes \eta\rangle=\langle K \xi, \eta\rangle, \quad \xi, \eta \in E_{\mathbb{C}} .
$$

In particular, $\tau(I)$ is a usual trace $\tau$, i.e. $\tau(I)=\tau$. It can be easily shown that

$$
\tau(K)=\sum_{\jmath=0}^{\infty}\left(K^{*} e_{\jmath}\right) \otimes e_{j},
$$

where $K^{*}$ is the adjoint operator of $K$. We note that the integral kernel operator $\Xi_{0,2}(\tau(K))$ associated with $\tau(K) \in\left(E_{\mathbb{C}} \otimes E_{\mathbb{C}}\right)^{*}$ is a continuous linear operator from $(E)$ into itself. 
Definition 3.1. For $K \in L\left(E_{\mathbb{C}}, E_{\mathbb{C}}^{*}\right)$, the $K$-Gross Laplacian $\Delta_{G}(K)$ is defined by the integral kernel operator given by

$$
\Delta_{G}(K)=\Xi_{0,2}(\tau(K))=\int_{T^{2}} \tau(K)(s, t) \partial_{s} \partial_{t} d s d t .
$$

In particular $\Delta_{G}(I)=\Delta_{G}$. If $f$ is a function such that $\xi \rightarrow f \xi$ gives a continuous operator $M_{f} \in L\left(E_{\mathbb{C}}, E_{\mathbb{C}}^{*}\right.$ ) (see [3]), then we see that the $M_{f^{-}}$ Gross Laplacian $\Delta_{G}\left(M_{f}\right)$ is given by

$$
\Delta_{G}\left(M_{f}\right)=\int_{T} f(t) \partial_{t}^{2} d t
$$

Moreover, if $K$ is the corresponding operator to $\kappa \in\left(E_{\mathbb{C}} \otimes E_{\mathbb{C}}\right)^{*}$ under the canonical isomorphism $\left(E_{\mathbb{C}} \otimes E_{\mathbb{C}}\right)^{*} \cong L\left(E_{\mathbb{C}}, E_{\mathbb{C}}^{*}\right)$, then we have $\Delta_{G}(K)=$ $\Xi_{0,2}(\kappa)$.

The following two lemmas are easily proved by direct computations.

Lemma 3.2. Let $K \in L\left(E_{\mathbb{C}}, E_{\mathbb{C}}^{*}\right)$. For any $\xi, \eta \in E_{\mathbb{C}}$,

$$
\widehat{\Delta_{G}(K)}(\xi, \eta)=\langle K \xi, \xi\rangle e^{\langle\xi, \eta\rangle}
$$

Lemma 3.3. Let $\phi(x)=\sum_{n=0}^{\infty}\left\langle: x^{\otimes n}:, f_{n}\right\rangle \in(E)$. Then for any $x \in E^{*}$, the series

$$
\sum_{k=0}^{\infty} \sum_{n=0}^{\infty}(n+2)(n+1)\left\langle: x^{\otimes n}:,\left(K^{*} e_{k} \otimes e_{k}\right) \widehat{\otimes}_{2} f_{n+2}\right\rangle
$$

absolutely converges.

THEOREM 3.4. Let $\phi(x)=\sum_{n=0}^{\infty}\left\langle: x^{\otimes n}:, f_{n}\right\rangle \in(E)$. Then for any $x \in$ $E^{*}$,

$$
\Delta_{G}(K) \phi(x)=\sum_{n=0}^{\infty}(n+2)(n+1)\left\langle: x^{\otimes n}:, \tau(K) \widehat{\otimes}_{2} f_{n+2}\right\rangle .
$$

Proof. From Lemma 3.3 we note that

$$
\sum_{k=0}^{\infty} D_{K^{*} e_{k}} D_{e_{k}} \phi(x)=\sum_{n=0}^{\infty}(n+2)(n+1)\left\langle: x^{\otimes n}:, \tau(K) \widehat{\otimes}_{2} f_{n+2}\right\rangle .
$$

Put $\Xi=\sum_{k=0}^{\infty} D_{K^{*} e_{k}} D_{e_{k}}$. Then by (3.1) and Lemma 3.2,

$$
\left.\left\langle\left\langle\Xi \phi_{\xi}, \phi_{\eta}\right\rangle\right\rangle=\left\langle\left\langle\left\langle\tau(K), \xi^{\otimes 2}\right\rangle \phi_{\xi}, \phi_{\eta}\right\rangle\right\rangle=\widehat{\Delta_{G}(K}\right)(\xi, \eta) .
$$


Hence $\Xi \phi(x)=\Delta_{G}(K) \phi(x)$ for any $\phi \in(E)$ and $x \in E^{*}$. This completes the proof.

Remark. For $M \in L\left(E_{\mathbb{C}}, E_{\mathbb{C}}\right)$, put $K=M^{*} M$. Then $K \in L\left(E_{\mathbb{C}}, E_{\mathbb{C}}^{*}\right)$. Hence by Theorem 3.4, the $K$-Gross Laplacian $\Delta_{G}(K)$ is given by

$$
\Delta_{G}(K)=\sum_{k=0}^{\infty} D_{M^{*} e_{k}}^{2} .
$$

Moreover, the symbol $\widehat{\Delta_{G}(K)}$ of $\Delta_{G}(K)$ is given by

$$
\Delta_{G}(K)(\xi, \eta)=\langle M \xi, M \xi\rangle e^{\langle\xi, \eta\rangle}, \quad \xi, \eta \in E_{\mathbb{C}}
$$

\section{$\S$ 4. $\mathcal{G}_{A, B}$ - and $\mathcal{F}_{A, B}$-transforms on white noise functionals}

Let $G L((E))$ (resp. $G L\left((E)^{*}\right)$ ) denote the group of all linear homeomorphisms from $(E)$ (resp. $\left.(E)^{*}\right)$ onto itself.

Lemma 4.1. For any $A \in L\left(E_{\mathbb{C}}, E_{\mathbb{C}}^{*}\right)$ and $B \in L\left(E_{\mathbb{C}}, E_{\mathbb{C}}\right)$, there exists an operator $\mathcal{G}_{A, B} \in L((E),(E))$ such that

$$
\mathcal{G}_{A, B} \phi_{\xi}=\phi_{B \xi} \exp \left\{\frac{\langle A \xi, \xi\rangle}{2}\right\}, \quad \xi \in E_{\mathbb{C}} .
$$

Proof. The proof follows from a simple application of Proposition 2.1.

Now we define operator-parameter $\mathcal{G}_{A, B}$-transforms $\left(A \in L\left(E_{\mathbb{C}}, E_{\mathbb{C}}^{*}\right)\right.$, $\left.B \in L\left(E_{\mathbb{C}}, E_{\mathbb{C}}\right)\right)$ as a generalization of scalar-parameter $\mathcal{G}_{\alpha, \beta}$-transforms $(\alpha, \beta \in \mathbb{C})$.

Definition 4.2. The operator $\mathcal{G}_{A, B} \in L((E),(E))$ obtained in Lemma 4.1 is called the $\mathcal{G}_{A, B}$-transform and the adjoint operator $\mathcal{G}_{A, B}^{*}$ of $\mathcal{G}_{A, B}$ is denoted by $\mathcal{F}_{A, B}$ and is called the $\mathcal{F}_{A, B}$-transform.

TheOrEm 4.3. Let $A, A^{\prime} \in L\left(E_{\mathbb{C}}, E_{\mathbb{C}}^{*}\right)$ and $B, B^{\prime} \in L\left(E_{\mathbb{C}}, E_{\mathbb{C}}\right)$. Then in order that $\mathcal{G}_{A^{\prime}, B^{\prime}} \mathcal{G}_{A, B} \phi=\phi$ for any $\phi \in(E)$, it is necessary and sufficient that

$$
B^{\prime} B=I \quad \text { and } \quad B^{*} A^{\prime} B+A=0 \quad \text { (zero operator). }
$$


Proof. By Lemma 4.1, we have, for any $\phi_{\xi} \in(E)$

$$
\begin{aligned}
\mathcal{G}_{A^{\prime}, B^{\prime}}\left(\mathcal{G}_{A, B} \phi_{\xi}\right) & =\phi_{B^{\prime} B \xi} \exp \left\{\frac{\left\langle A^{\prime} B \xi, B \xi\right\rangle+\langle A \xi, \xi\rangle}{2}\right\} \\
& =\phi_{B^{\prime} B \xi} \exp \left\{\frac{\left\langle\left(B^{*} A^{\prime} B+A\right) \xi, \xi\right\rangle}{2}\right\} \\
& =\mathcal{G}_{B^{*} A^{\prime} B+A, B^{\prime} B} \phi_{\xi} .
\end{aligned}
$$

Hence we complete the proof.

By duality we have the following theorem.

Theorem 4.4. Let $A, A^{\prime} \in L\left(E_{\mathbb{C}}, E_{\mathbb{C}}^{*}\right)$ and $B, B^{\prime} \in L\left(E_{\mathbb{C}}, E_{\mathbb{C}}\right)$. Then in order that $\mathcal{F}_{A^{\prime}, B^{\prime}} \mathcal{F}_{A, B} \Phi=\Phi$ for any $\Phi \in(E)^{*}$, it is necessary and sufficient that

$$
B B^{\prime}=I \quad \text { and } \quad B^{\prime *} A B^{\prime}+A^{\prime}=0 \quad \text { (zero operator) }
$$

Remark. $\left\{\mathcal{G}_{A, B} \mid A \in L\left(E_{\mathbb{C}}, E_{\mathbb{C}}^{*}\right), B \in G L\left(E_{\mathbb{C}}\right)\right\}$ (resp. $\left\{\mathcal{F}_{A, B} \mid A \in\right.$ $\left.\left.L\left(E_{\mathbb{C}}, E_{\mathbb{C}}^{*}\right), B \in G L\left(E_{\mathbb{C}}\right)\right\}\right)$ is a subgroup of $G L((E))$ (resp. $\left.G L\left((E)^{*}\right)\right)$.

The following result shows that the $\mathcal{G}_{A, B}$-transform has the integral representation.

Proposition 4.5. Let $A \in L\left(E_{\mathbb{C}}, E_{\mathbb{C}}^{*}\right)$ and $B, C \in L\left(E_{\mathbb{C}}, E_{\mathbb{C}}\right)$ such that $C^{*} C=A-B^{*} B+I$. Then for any $\phi \in(E), \mathcal{G}_{A, B} \phi$ is represented by

$$
\mathcal{G}_{A, B} \phi(x)=\int_{E^{*}} \phi\left(C^{*} y+B^{*} x\right) d \mu(y), \quad x \in E^{*} .
$$

Proof. It is known [4] that the linear operator $\phi \rightarrow \int_{E^{*}} \phi\left(C^{*} y+\right.$ $\left.B^{*} x\right) d \mu(y)$ is a continuous from $(E)$ into itself. Hence it suffices to show that (4.1) holds for all exponential vectors $\phi_{\xi}$ since $\left\{\phi_{\xi}: \xi \in E_{\mathbb{C}}\right\}$ spans a dense subspace of $(E)$. Note that $\phi_{\xi}$ is given by

$$
\phi_{\xi}(x)=\exp \left\{\langle x, \xi\rangle-\frac{1}{2}\langle\xi, \xi\rangle\right\}
$$


Using the fact that $\int_{E^{*}} e^{\langle y, A \xi\rangle} d \mu(y)=e^{\langle A \xi, A \xi\rangle / 2}$, we have

$$
\begin{aligned}
\int_{E^{*}} \phi_{\xi}\left(C^{*} y+B^{*} x\right) d \mu(y) & =\phi_{B \xi}(x) \exp \left\{\frac{\langle C \xi, C \xi\rangle+\langle B \xi, B \xi\rangle-\langle\xi, \xi\rangle}{2}\right\} \\
& =\phi_{B \xi}(x) \exp \left\{\frac{\langle A \xi, \xi\rangle}{2}\right\} \\
& =\mathcal{G}_{A, B} \phi_{\xi}(x) .
\end{aligned}
$$

Thus we complete the proof.

The following theorem gives a characterization for the $\mathcal{F}_{A, B}$-transforms.

TheOrem 4.6. Let $A \in L\left(E_{\mathbb{C}}, E_{\mathbb{C}}^{*}\right)$ and $B \in L\left(E_{\mathbb{C}}, E_{\mathbb{C}}\right)$. Then $\mathcal{F}_{A, B^{-}}$ transform is the unique operator in $L\left((E)^{*},(E)^{*}\right)$ such that for each $\Phi \in$ $(E)^{*}$, the $S$-transform of $\mathcal{F}_{A, B} \Phi$ is given by

$$
S\left(\mathcal{F}_{A, B} \Phi\right)(\xi)=S \Phi(B \xi) \exp \left\{\frac{\langle A \xi, \xi\rangle}{2}\right\}, \quad \xi \in E_{\mathbb{C}}
$$

Proof. Let $\Phi \in(E)^{*}$ and $\xi \in E_{\mathbb{C}}$. Then by the definition of $\mathcal{F}_{A, B}$,

$$
S\left(\mathcal{F}_{A, B} \Phi\right)(\xi)=\left\langle\left\langle\mathcal{F}_{A, B} \Phi, \phi_{\xi}\right\rangle\right\rangle=\left\langle\left\langle\mathcal{G}_{A, B}^{*} \Phi, \phi_{\xi}\right\rangle\right\rangle=\left\langle\left\langle\Phi, \mathcal{G}_{A, B} \phi_{\xi}\right\rangle\right\rangle .
$$

Hence by Lemma 4.1, we have

$$
S\left(\mathcal{F}_{A, B} \Phi\right)(\xi)=\left\langle\left\langle\Phi, \phi_{B \xi}\right\rangle\right\rangle \exp \left\{\frac{\langle A \xi, \xi\rangle}{2}\right\}=S \Phi(B \xi) \exp \left\{\frac{\langle A \xi, \xi\rangle}{2}\right\},
$$

so that we complete the proof.

We now obtain an explicit expression of $\mathcal{F}_{A, B} \Phi$.

TheOrEm 4.7. Let $A \in L\left(E_{\mathbb{C}}, E_{\mathbb{C}}^{*}\right), B \in L\left(E_{\mathbb{C}}, E_{\mathbb{C}}\right)$ and let $\Phi \in(E)^{*}$ be given by

$$
\Phi(x)=\sum_{n=0}^{\infty}\left\langle: x^{\otimes n}:, F_{n}\right\rangle, \quad F_{n} \in\left(E_{\mathbb{C}}^{\otimes n}\right)_{\mathrm{sym}}^{*} .
$$

Then $\mathcal{F}_{A, B} \Phi$ is given by

$$
\mathcal{F}_{A, B} \Phi(x)=\sum_{n=0}^{\infty}\left\langle: x^{\otimes n}:, \sum_{m=0}^{[n / 2]} \frac{1}{m ! 2^{m}}\left(\left(B^{*}\right)^{\otimes(n-2 m)} F_{n-2 m}\right) \widehat{\otimes} \tau(A)^{\otimes m}\right\rangle .
$$


Proof. Since $S(\Phi)(\xi)=\sum_{n=0}^{\infty}\left\langle F_{n}, \xi^{\otimes n}\right\rangle$, we have, by Theorem 4.6

$$
\begin{aligned}
S\left(\mathcal{F}_{A, B} \Phi\right)(\xi) & =S \Phi(B \xi) \exp \left\{\frac{\langle A \xi, \xi\rangle}{2}\right\} \\
& =\sum_{n=0}^{\infty} \sum_{m=0}^{\infty} \frac{1}{m ! 2^{m}}\left\langle F_{n},(B \xi)^{\otimes n}\right\rangle\langle A \xi, \xi\rangle^{m} \\
& =\sum_{n=0}^{\infty} \sum_{m=0}^{\infty} \frac{1}{m ! 2^{m}}\left\langle\left(\left(B^{*}\right)^{\otimes n} F_{n}\right) \widehat{\otimes} \tau(A)^{\otimes m}, \xi^{\otimes(n+2 m)}\right\rangle \\
& =\sum_{n=0}^{\infty} \sum_{m=0}^{[n / 2]} \frac{1}{m ! 2^{m}}\left\langle\left(\left(B^{*}\right)^{\otimes(n-2 m)} F_{n-2 m}\right) \widehat{\otimes} \tau(A)^{\otimes m}, \xi^{\otimes n}\right\rangle .
\end{aligned}
$$

Hence we obtain the desired expression of $\mathcal{F}_{A, B} \Phi$.

The following theorem gives an expression of the $\mathcal{G}_{A, B}$-transforms.

Theorem 4.8. Let $A \in L\left(E_{\mathbb{C}}, E_{\mathbb{C}}^{*}\right), B \in L\left(E_{\mathbb{C}}, E_{\mathbb{C}}\right)$ and $\phi \in(E)$ be given by

$$
\phi(x)=\sum_{n=0}^{\infty}\left\langle: x^{\otimes n}:, f_{n}\right\rangle, \quad f_{n} \in E_{\mathbb{C}}^{\widehat{\otimes} n} .
$$

Then $\mathcal{G}_{A, B} \phi$ is given by

$$
\mathcal{G}_{A, B} \phi(x)=\sum_{n=0}^{\infty}\left\langle: x^{\otimes n}:, \sum_{m=0}^{\infty} \frac{(n+2 m) !}{n ! m ! 2^{m}} B^{\otimes n}\left(\tau(A)^{\otimes m} \widehat{\otimes}_{2 m} f_{n+2 m}\right)\right\rangle .
$$

Proof. For any $\Phi \in(E)^{*}$ with $\Phi(x)=\sum_{n=0}^{\infty}\left\langle: x^{\otimes n}:, F_{n}\right\rangle$, we have

$$
\begin{aligned}
\left\langle\left\langle\Phi, \mathcal{G}_{A, B} \phi\right\rangle\right\rangle & =\left\langle\left\langle\mathcal{F}_{A, B} \Phi, \phi\right\rangle\right\rangle \\
& =\sum_{n=0}^{\infty} n !\left\langle\sum_{m=0}^{[n / 2]} \frac{1}{m ! 2^{m}}\left(\left(B^{*}\right)^{\otimes(n-2 m)} F_{n-2 m}\right) \widehat{\otimes} \tau(A)^{\otimes m}, f_{n}\right\rangle \\
& =\sum_{n=0}^{\infty} \sum_{m=0}^{\infty} \frac{(n+2 m) !}{m ! 2^{m}}\left\langle\left(\left(B^{*}\right)^{\otimes n} F_{n}\right) \widehat{\otimes} \tau(A)^{\otimes m}, f_{n+2 m}\right\rangle \\
& =\sum_{n=0}^{\infty} n !\left\langle F_{n}, \sum_{m=0}^{\infty} \frac{(n+2 m) !}{n ! m ! 2^{m}} B^{\otimes n}\left(\tau(A)^{\otimes m} \widehat{\otimes}_{2 m} f_{n+2 m}\right)\right\rangle .
\end{aligned}
$$

Hence we obtain the desired expression of $\mathcal{G}_{A, B} \phi$. 
Theorem 4.9. Let $A \in L\left(E_{\mathbb{C}}, E_{\mathbb{C}}^{*}\right)$ and $B \in L\left(E_{\mathbb{C}}, E_{\mathbb{C}}\right)$. Then the symbols of $\mathcal{G}_{A, B}$ and $\mathcal{F}_{A, B}$ are given by

$$
\begin{aligned}
& \widehat{\mathcal{G}_{A, B}}(\xi, \eta)=\exp \left\{\frac{\langle A \xi, \xi\rangle}{2}+\langle B \xi, \eta\rangle\right\} \\
& \widehat{\mathcal{F}_{A, B}}(\xi, \eta)=\widehat{\mathcal{G} A, B}(\eta, \xi)=\exp \left\{\frac{\langle A \eta, \eta\rangle}{2}+\langle B \eta, \xi\rangle\right\} .
\end{aligned}
$$

Further, the Fock expansion of $\mathcal{G}_{A, B}$ and $\mathcal{F}_{A, B}$ are given by

$$
\begin{aligned}
& \mathcal{G}_{A, B}=\sum_{l, m=0}^{\infty} \frac{1}{l ! m ! 2^{m}} \Xi_{l, l+2 m}\left(\tau(B-I, l) \otimes \tau(A)^{\otimes m}\right) \\
& \mathcal{F}_{A, B}=\sum_{l, m=0}^{\infty} \frac{1}{l ! m ! 2^{l}} \Xi_{2 l+m, m}\left(\tau(A)^{\otimes l} \otimes \tau(B-I, m)\right),
\end{aligned}
$$

where $\tau(B-I, l) \in\left(E^{\otimes 2 l}\right)^{*}$ is given by

$$
\tau(B-I, l)=\sum_{i_{1}, \cdots, l_{l}=0}^{\infty}\left(B^{*}-I\right) e_{i_{1}} \otimes \cdots \otimes\left(B^{*}-I\right) e_{i_{l}} \otimes e_{i_{1}} \otimes \cdots \otimes e_{i_{l}} .
$$

Proof. By the definition of symbol of operator, we have

$$
\widehat{\mathcal{G} A, B}(\xi, \eta)=\exp \left\{\frac{\langle A \xi, \xi\rangle}{2}+\langle B \xi, \eta\rangle\right\} .
$$

Thus we have

$$
e^{-\langle\xi, \eta\rangle} \widehat{\mathcal{G}_{A, B}}(\xi, \eta)=\exp \left\{\frac{\langle A \xi, \xi\rangle}{2}+\langle(B-I) \xi, \eta\rangle\right\} .
$$

Hence by using the Taylor expansion, we obtain

$$
\mathcal{G}_{A, B}=\sum_{l, m=0}^{\infty} \frac{1}{l ! m ! 2^{m}} \Xi_{l, l+2 m}\left(\tau(B-I, l) \otimes \tau(A)^{\otimes m}\right) .
$$

The rest of proof follows from the duality.

\section{§5. Characterizations of $\mathcal{G}_{A, B^{-}}$and $\mathcal{F}_{A, B}$-transforms}

Let $D_{\eta}$ denote the Gateaux differential operator in the direction $\eta \in E^{*}$. Then $D_{\eta} \in L((E),(E))$ and $D_{\eta}^{*} \in L\left((E)^{*},(E)^{*}\right)$ for any $\eta \in E$. For each $\eta \in E$, let $q_{\eta}$ denote the multiplication by $\langle\cdot, \eta\rangle$. Then $q_{\eta} \in L((E),(E))$ for 
any $\eta \in E$. For $\eta \in E$, the operators $D_{\eta}$ and $q_{\eta}$ are continuously extended to operators in $L\left((E)^{*},(E)^{*}\right)$, which are denoted by $\widetilde{D_{\eta}}$ and $\widetilde{q_{\eta}}$, respectively. Moreover, we have $\widetilde{q}_{\eta}=\widetilde{D_{\eta}}+D_{\eta}^{*}$.

In [7], Hida, Kuo and Obata proved a characterization theorem for the Fourier-Mehler transform in terms of the operators $\widetilde{D_{\eta}}$ and $\widetilde{q_{\eta}}$. In [1], Chung and $\mathrm{Ji}$ proved a characterization theorem for the $\mathcal{F}_{\alpha, \beta}$-transform in terms of the operators $\widetilde{D_{\eta}}$ and $\widetilde{q_{\eta}}$. In this section, we extend some results in [1] for the operator-parameter $\mathcal{G}_{A, B^{-}}$and $\mathcal{F}_{A, B^{-}}$-transforms.

Definition 5.1. For each $y \in E^{*}$ the translation operator $T_{y}$ is defined by

$$
T_{y} \phi(x)=\phi(x+y), \quad \phi \in(E), x \in E^{*} .
$$

Note that the operator $T_{y}$ is a continuous linear operator from $(E)$ into itself (see [21]).

Theorem 5.2. Let $A \in L\left(E_{\mathbb{C}}, E_{\mathbb{C}}^{*}\right)$ and $B \in G L\left(E_{\mathbb{C}}\right)$. Then the $\mathcal{G}_{A, B^{-}}$ transform has the following properties:

(i) $\mathcal{G}_{A, B} D_{y}=D_{B^{-1 *}{ }_{y}} \mathcal{G}_{A, B}, y \in E^{*}$,

(ii) $\mathcal{G}_{A, B} D_{\eta}^{*}=D_{\left(B-\frac{1}{2} B^{-1 *}\left(A+A^{*}\right)\right) \eta}^{*} \mathcal{G}_{A, B}+q_{\frac{1}{2} B^{-1 *}\left(A+A^{*}\right) \eta} \mathcal{G}_{A, B}, \eta \in E$,

(iii) $\mathcal{G}_{A, B} q_{\eta}=D_{\left(B-\frac{1}{2} B^{-1 *}\left(A+A^{*}\right)-B^{-1 *}\right) \eta}^{*} \mathcal{G}_{A, B}+q_{\left(\frac{1}{2} B^{-1 *}\left(A+A^{*}\right)+B^{-1 *}\right) \eta} \mathcal{G}_{A, B}$,

(iv) $\mathcal{G}_{A, B} T_{y}=T_{B^{-1}{ }_{y}} \mathcal{G}_{A, B}, y \in E^{*}$,

(v) $\mathcal{G}_{A, B} \Delta_{G}(K)=\Delta_{G}\left(B^{-1^{*}} K B^{-1}\right) \mathcal{G}_{A, B}$,

(vi) $\mathcal{G}_{A, B} N=N \mathcal{G}_{A, B}+\Delta_{G}\left(B^{-1^{*}} A B^{-1}\right) \mathcal{G}_{A, B}$.

Proof. Since $\left\{\phi_{\xi}: \xi \in E_{\mathbb{C}}\right\}$ spans a dense subspace of $(E)$ and the operators $\mathcal{G}_{A, B}, D_{y}, T_{y}, N$ and $\Delta_{G}(K)$ are continuous linear operators from $(E)$ into itself, it is sufficient to prove that for any $\phi_{\xi}$ with $\xi \in E_{\mathbb{C}}$, the properties (i)-(vi) hold. Let $\xi \in E_{\mathbb{C}}$. Then

(i) Since for any $y \in E^{*}, D_{y} \phi_{\xi}=\langle y, \xi\rangle \phi_{\xi}$, by Lemma 4.1, we have

$$
\mathcal{G}_{A, B} D_{y} \phi_{\xi}=\langle y, \xi\rangle \phi_{B \xi} \exp \left\{\frac{\langle A \xi, \xi\rangle}{2}\right\}
$$


and

$$
D_{y} \mathcal{G}_{A, B} \phi_{\xi}=\langle y, B \xi\rangle \phi_{B \xi} \exp \left\{\frac{\langle A \xi, \xi\rangle}{2}\right\}
$$

Hence we have

$$
\mathcal{G}_{A, B} D_{y} \phi_{\xi}=D_{B^{-1 *}{ }^{*}} \mathcal{G}_{A, B} \phi_{\xi}
$$

(ii) By applying Theorem 4.8 and using the relation:

$$
\begin{aligned}
& (n+2 l+1) \tau(A)^{\otimes l} \widehat{\otimes}_{2 l}\left(\eta \widehat{\otimes} \xi^{\otimes(n+2 l)}\right) \\
& \quad=2 l\left\langle\left(\frac{A+A^{*}}{2}\right) \eta, \xi\right\rangle\langle A \xi, \xi\rangle^{(l-1)} \xi^{\otimes(n+1)}+(n+1)\langle A \xi, \xi\rangle^{l} \eta \widehat{\otimes} \xi^{\otimes n}
\end{aligned}
$$

we obtain

$$
\begin{aligned}
& \mathcal{G}_{A, B} D_{\eta}^{*} \phi_{\xi}(x) \\
& =\mathcal{G}_{A, B}\left(\sum_{n=0}^{\infty} \frac{1}{n !}\left\langle: x^{\otimes(n+1)}:, \eta \widehat{\otimes} \xi^{\otimes n}\right\rangle\right)(x) \\
& =\sum_{n=0}^{\infty}\left\langle: x^{\otimes(n+1)}:, \sum_{l=0}^{\infty} \frac{(n+2 l+1) !}{(n+1) ! l ! 2^{l}} B^{\otimes(n+1)} \tau(A)^{\otimes l} \widehat{\otimes}_{2 l} \frac{\eta \widehat{\otimes}^{\otimes(n+2 l)}}{(n+2 l) !}\right\rangle \\
& +\sum_{l=1}^{\infty} \frac{2 l}{l ! 2^{l}} \tau(A)^{\otimes l} \widehat{\otimes}_{2 l}\left(\eta \widehat{\otimes} \xi^{\otimes(2 l-1)}\right) \\
& =\sum_{n=0}^{\infty}\left\langle: x^{\otimes(n+1)}:, \sum_{l=0}^{\infty} \frac{n+1}{(n+1) ! l ! 2^{l}}\langle A \xi, \xi\rangle^{l} B^{\otimes(n+1)}\left(\eta \widehat{\otimes} \xi^{\otimes n}\right)\right\rangle \\
& +\sum_{n=0}^{\infty}\left\langle: x^{\otimes(n+1)}:\right. \\
& \left.\sum_{l=0}^{\infty} \frac{2 l}{(n+1) ! l ! 2^{l}} \frac{1}{2}\left\langle\left(A+A^{*}\right) \eta, \xi\right\rangle\langle A \xi, \xi\rangle^{l-1} B^{\otimes(n+1)} \xi^{\otimes(n+1)}\right\rangle \\
& +\sum_{l=0}^{\infty} \frac{1}{l ! 2^{l}} \tau(A)^{\otimes(l+1)} \widehat{\otimes}_{2(l+1)}\left(\eta \widehat{\otimes} \xi^{\otimes(2 l+1)}\right) \\
& =\sum_{n=0}^{\infty}\left\langle: x^{\otimes(n+1)}: \frac{B^{\otimes(n+1)}\left(\eta \widehat{\otimes} \xi^{\otimes n}\right)}{n !}\right\rangle \exp \left\{\frac{\langle A \xi, \xi\rangle}{2}\right\} \\
& +\frac{1}{2}\left\langle\left(A+A^{*}\right) \eta, \xi\right\rangle \sum_{n=0}^{\infty}\left\langle: x^{\otimes n}: \frac{B^{\otimes n} \xi^{\otimes n}}{n !}\right\rangle \exp \left\{\frac{\langle A \xi, \xi\rangle}{2}\right\} \\
& =D_{B \eta}^{*} \mathcal{G}_{A, B} \phi_{\xi}(x)+\frac{1}{2}\left\langle\left(A+A^{*}\right) \eta, \xi\right\rangle \mathcal{G}_{A, B} \phi_{\xi}(x) \text {. }
\end{aligned}
$$


Since $q_{\eta}=D_{\eta}+D_{\eta}^{*}, \mathcal{G}_{A, B} D_{\eta}^{*} \phi_{\xi}=D_{\left(B-\frac{1}{2} B^{-1^{*}}\left(A+A^{*}\right)\right) \eta}^{*} \mathcal{G}_{A, B} \phi_{\xi}+q_{\frac{1}{2} B^{-1 *}\left(A+A^{*}\right) \eta}$ $\mathcal{G}_{A, B} \phi_{\xi}$. We complete the proof.

(iii) Note that $q_{\eta}=D_{\eta}+D_{\eta}^{*}$. So by (i) and (ii) we have

$$
\mathcal{G}_{A, B} q_{\eta}=D_{\left(B-\frac{1}{2} B^{-1 *}\left(A+A^{*}\right)-B^{-1 *}\right) \eta}^{*} \mathcal{G}_{A, B}+q_{\left(\frac{1}{2} B^{-1 *}\left(A+A^{*}\right)+B^{-1 *}\right) \eta} \mathcal{G}_{A, B} .
$$

(iv) We note that for any $y \in E^{*}, T_{y} \phi_{\xi}=\exp \{\langle y, \xi\rangle\} \phi_{\xi}$. So we obtain

$$
\mathcal{G}_{A, B} T_{y} \phi_{\xi}=\phi_{B \xi} \exp \left\{\frac{\langle A \xi, \xi\rangle}{2}\right\} \exp \{\langle y, \xi\rangle\}=T_{B^{-1}{ }^{*}} \mathcal{G}_{A, B} \phi_{\xi}
$$

(v) We note that $\Delta_{G}(K) \phi_{\xi}=\langle K \xi, \xi\rangle \phi_{\xi}$. So we have

$$
\mathcal{G}_{A, B} \Delta_{G}(K) \phi_{\xi}=\langle K \xi, \xi\rangle \phi_{B \xi} \exp \left\{\frac{\langle A \xi, \xi\rangle}{2}\right\}=\Delta_{G}\left(B^{-1^{*}} K B^{-1}\right) \mathcal{G}_{A, B} \phi_{\xi} .
$$

(vi) We note that $N \phi_{\xi}(x)=\sum_{n=0}^{\infty} n\left\langle: x^{\otimes n}:, \xi^{\otimes n} / n !\right\rangle$. So by Theorem 4.8 , we obtain

$$
\begin{aligned}
\mathcal{G}_{A, B} N \phi_{\xi} & =N \phi_{B \xi} \exp \left\{\frac{\langle A \xi, \xi\rangle}{2}\right\}+\langle A \xi, \xi\rangle \phi_{B \xi} \exp \left\{\frac{\langle A \xi, \xi\rangle}{2}\right\} \\
& =N \mathcal{G}_{A, B} \phi_{\xi}+\Delta_{G}\left(B^{-1^{*}} A B^{-1}\right) \mathcal{G}_{A, B} \phi_{\xi}
\end{aligned}
$$

This completes the proof.

Now we will prove a characterization theorem for the $\mathcal{G}_{A, B}$-transform. For the proof we adopt the method used in $[1,7]$.

TheOREm 5.3. Let $A \in L\left(E_{\mathbb{C}}, E_{\mathbb{C}}^{*}\right)$ and $B \in G L\left(E_{\mathbb{C}}\right)$. Suppose $\mathcal{A}_{A, B}$ is a linear operator in $L((E),(E))$ satisfying the properties (ii) and (iii) in Theorem 5.2. Then $\mathcal{A}_{A, B}$ is a constant multiple of $\mathcal{G}_{A, B}$.

For the proof we need the following proposition.

Proposition 5.4. ([7]) Let $L \in L((E),(E))$ satisfy

(i) $L q_{\eta}=q_{\eta} L$ for every $\eta \in E$;

(ii) $L D_{\eta}=D_{\eta} L$ for every $\eta \in E$.

Then $L$ is a scalar operator. 
Proof of Theorem 5.3. Suppose $\mathcal{A}_{A, B}$ is in $L((E),(E))$ and satisfies the properties (ii) and (iii) in Theorem 5.2. Let $A^{\prime} \in L\left(E_{\mathbb{C}}, E_{\mathbb{C}}^{*}\right)$ and $B^{\prime} \in$ $L\left(E_{\mathbb{C}}, E_{\mathbb{C}}\right)$ satisfy the following equations:

$$
B^{\prime} B=I \quad \text { and } \quad B^{*} A^{\prime} B+A=0 \quad \text { (zero operator). }
$$

Then, using the equations (ii), (iii) in Theorem 5.2 and (5.1), we obtain

$$
\left(\mathcal{G}_{A^{\prime}, B^{\prime}} \mathcal{A}_{A, B}\right) D_{\eta}=D_{\eta} \mathcal{G}_{A^{\prime}, B^{\prime}} \mathcal{A}_{A, B}
$$

Next using (ii) and (iii) in Theorem 5.2 and the equations (5.1) again, we obtain

$$
\left(\mathcal{G}_{A^{\prime}, B^{\prime}} \mathcal{A}_{A, B}\right) D_{\eta}^{*}=D_{\eta}^{*} \mathcal{G}_{A^{\prime}, B^{\prime}} \mathcal{A}_{A, B}
$$

Note that $D_{\eta}=q_{\eta}-D_{\eta}^{*}$. Using (5.2) and (5.3), we have

$$
\mathcal{G}_{A^{\prime}, B^{\prime}} \mathcal{A}_{A, B} q_{\eta}=q_{\eta} \mathcal{G}_{A^{\prime}, B^{\prime}} \mathcal{A}_{A, B}
$$

Hence by Proposition 5.4, $\mathcal{G}_{A^{\prime}, B^{\prime}} \mathcal{A}_{A, B}$ is a scalar operator. Equivalently, $\mathcal{A}_{A, B}$ is a constant multiple of the $\mathcal{G}_{A, B}$-transform.

By duality argument for Theorems 5.2 and 5.3, we obtain a characterization theorem of the $\mathcal{F}_{A, B}$-transform.

Theorem 5.5. Let $A, K \in L\left(E_{\mathbb{C}}, E_{\mathbb{C}}^{*}\right)$ and $B \in G L\left(E_{\mathbb{C}}\right)$. Then the $\mathcal{F}_{A, B}$-transform has the following properties:

(i) $\mathcal{F}_{A, B} D_{y}^{*}=D_{B^{*} y}^{*} \mathcal{F}_{A, B}, y \in E^{*}$,

(ii) $\mathcal{F}_{A, B} \widetilde{D}_{\eta}=\widetilde{D}_{\left(B^{-1}+\frac{1}{2}\left(A+A^{*}\right) B^{-1}\right) \eta} \mathcal{F}_{A, B}-q_{\frac{1}{2}\left(A+A^{*}\right) B^{-1} \eta} \mathcal{F}_{A, B}, \eta \in E$,

(iii) $\mathcal{F}_{A, B} \widetilde{q}_{\eta}=\widetilde{D}_{\left(B^{-1}+\frac{1}{2}\left(A+A^{*}\right) B^{-1}-B^{*}\right) \eta} \mathcal{F}_{A, B}+q_{\left(-\frac{1}{2}\left(A+A^{*}\right) B^{-1}+B^{*}\right) \eta} \mathcal{F}_{A, B}$, $\eta \in E$,

(iv) $\mathcal{F}_{A, B}\left(\Delta_{G}(K)\right)^{*}=\left(\Delta_{G}\left(B^{*} K B\right)\right)^{*} \mathcal{F}_{A, B}$,

(v) $\mathcal{F}_{A, B} N=N \mathcal{F}_{A, B}-\left(\Delta_{G}(A)\right)^{*} \mathcal{F}_{A, B}$.

Theorem 5.6. Let $A \in L\left(E_{\mathbb{C}}, E_{\mathbb{C}}^{*}\right)$ and $B \in G L\left(E_{\mathbb{C}}\right)$. Suppose $\mathcal{A}_{A, B}$ is a linear operator in $L\left((E)^{*},(E)^{*}\right)$ satisfying the properties (ii) and (iii) in Theorem 5.5. Then $\mathcal{A}_{A, B}$ is a constant multiple of $\mathcal{F}_{A, B}$. 


\section{$\S$ 6. Applications to Cauchy Problems}

A one-parameter subgroup $\left\{V_{\theta}\right\}_{\theta \in \mathbb{R}}$ of $G L((E))$ is called differentiable if for every $\phi \in(E)$,

$$
\lim _{\theta \rightarrow 0} \frac{V_{\theta} \phi-\phi}{\theta}
$$

converges in the topology of $(E)$. In this case, a linear operator $X$ from $(E)$ into itself defined by

$$
X \phi=\lim _{\theta \rightarrow 0} \frac{V_{\theta} \phi-\phi}{\theta}, \quad \phi \in(E)
$$

is called the infinitesimal generator of $\left\{V_{\theta}\right\}_{\theta \in \mathbb{R}}$. It is shown that $X \in$ $L((E),(E))$ and that for any $\theta \in \mathbb{R}$ and $\phi \in(E)$, we have

$$
V_{\theta} X \phi=X V_{\theta} \phi=\lim _{\epsilon \rightarrow 0} \frac{V_{\theta+\epsilon} \phi-V_{\theta} \phi}{\epsilon}
$$

where the right hand side converges in the topology of $(E)$.

Definition 6.1. A differentiable one-parameter group $\left\{V_{\theta}\right\}_{\theta \in \mathbb{R}}$ with the infinitesimal generator $X$ is called regular if for any $p \geq 0$, there exists $q \geq 0$ such that

$$
\lim _{\theta \rightarrow 0} \sup _{\|\phi\|_{q} \leq 1}\left\|\frac{V_{\theta} \phi-\phi}{\theta}-X \phi\right\|_{p}=0
$$

In this section we investigate the following Cauchy problem:

$$
\frac{\partial u(x, \theta)}{\partial \theta}=\Delta_{G}(K) u(x, \theta), \quad u(x, 0)=\phi(x),
$$

where $\Delta_{G}(K)$ is the $K$-Gross Laplacian and $\phi \in(E)$.

We easily see that $\left\{\mathcal{G}_{2 \theta K, I}\right\}_{\theta \in \mathbb{R}}$ is a one-parameter subgroup of $G L((E))$. The following theorem shows that for any $K \in L\left(E_{\mathbb{C}}, E_{\mathbb{C}}^{*}\right),\left\{\mathcal{G}_{2 \theta K, I}\right\}_{\theta \in \mathbb{R}}$ is differentiable. For the proof, we will adopt the method used in $[1,18]$.

THEOREM 6.2. Let $K \in L\left(E_{\mathbb{C}}, E_{\mathbb{C}}^{*}\right)$. Then $\left\{\mathcal{G}_{2 \theta K, I}\right\}_{\theta \in \mathbb{R}}$ is a differentiable one-parameter subgroup of $G L((E))$ whose infinitesimal generator is $\Delta_{G}(K)$. Moreover, $\left\{\mathcal{G}_{2 \theta K, I}\right\}_{\theta \in \mathbb{R}}$ is regular. 
Proof. Note that $\left\{\mathcal{G}_{2 \theta K, I}\right\}_{\theta \in \mathbb{R}}$ is a one-parameter subgroup of $G L((E))$. Now we consider the operator symbol $\widehat{\mathcal{G}}_{2 \theta K, I}$ of $\mathcal{G}_{2 \theta K, I}$. For any $\xi, \eta \in E_{\mathbb{C}}$, we put

$$
f(\theta)=\widehat{\mathcal{G}}_{2 \theta K, I}(\xi, \eta)=\exp \{\theta\langle K \xi, \xi\rangle+\langle\xi, \eta\rangle\} .
$$

Then we have

$$
f^{\prime}(\theta)=\langle K \xi, \xi\rangle f(\theta) \quad \text { and } \quad f^{\prime \prime}(\theta)=\langle K \xi, \xi\rangle^{2} f(\theta) .
$$

Let $\theta_{0}>0$ be fixed. Whenever $|\theta| \leq \theta_{0},\left|f^{\prime \prime}(\theta)\right| \leq \exp \left\{\left(\theta_{0}+1\right)|\langle K \xi, \xi\rangle|+\right.$ $|\langle\xi, \eta\rangle|\}$. Now we put $g_{\theta}(\xi, \eta)=f(\theta)-f(0)-f^{\prime}(0) \theta$. Then by the Taylor theorem, we have $\left|g_{\theta}(\xi, \eta)\right| \leq|\theta|^{2} / 2 \max _{|\theta| \leq \theta_{0}}\left|f^{\prime \prime}(\theta)\right|$. Hence for any $p \geq 0$ and $\epsilon>0$, there exists $q=q\left(K, p, \epsilon, \theta_{0}\right) \geq 0$ such that

$$
\left.\left|g_{\theta}(\xi, \eta)\right| \leq \frac{|\theta|^{2}}{2} \exp \left\{\epsilon\left(|\xi|^{2}\right)_{p+q}+|\eta|_{-p}^{2}\right)\right\}, \quad|\theta| \leq \theta_{0}, \quad \xi, \eta \in E_{\mathbb{C}} .
$$

It then follows from Proposition 2.1 that there exists $\Xi_{\theta} \in L((E),(E))$ such that $\widehat{\Xi}_{\theta}=g_{\theta}$ and

$$
\left\|\Xi_{\theta} \phi\right\|_{p-1} \leq \frac{|\theta|^{2}}{2} M(\epsilon, q, r)\|\phi\|_{p+q+r+1}, \quad \phi \in(E)
$$

for some $r \geq 0$. On the other hand, by Lemma 3.2, we see that

$$
\begin{aligned}
f(0) & =e^{\langle\xi, \eta\rangle}=\widehat{I}(\xi, \eta) \\
f^{\prime}(0) & =\langle K \xi, \xi\rangle e^{\langle\xi, \eta\rangle}=\Delta_{G(K)}(\xi, \eta) .
\end{aligned}
$$

Hence we have

$$
\Xi_{\theta}=\mathcal{G}_{2 \theta K, I}-I-\theta \Delta_{G}(K) .
$$

From (6.2), it follows that

$$
\sup _{\|\phi\|_{p+q+r+1} \leq 1}\left\|\frac{\mathcal{G}_{2 \theta K, I} \phi-\phi}{\theta}-\Delta_{G}(K) \phi\right\|_{p-1} \leq \frac{|\theta|}{2} C M(\epsilon, q, r) \rightarrow 0
$$

as $\theta \rightarrow 0$. Hence we complete the proof.

Theorem 6.3. Let $K \in L\left(E_{\mathbb{C}}, E_{\mathbb{C}}^{*}\right)$ and $\phi \in(E)$. Put $u(x, \theta)=$ $\mathcal{G}_{2 \theta K, I} \phi(x)$. Then we have 
(1) $u(\cdot, \theta) \in(E)$ for any $\theta \in \mathbb{R}$.

(2) $u(\cdot, \theta)$ is differentiable with respect to $\theta$ in the topology of $(E)$.

(3) $u(x, \theta)$ is a unique solution of (6.1) such that $u(x, \theta)$ satisfy (1) and (2).

(4) If there exists $C \in L\left(E_{\mathbb{C}}, E_{\mathbb{C}}\right)$ such that $K=C^{*} C$, then $u(x, \theta)$ is represented by an integral:

$$
u(x, \theta)=\int_{E^{*}} \phi\left((2 \theta)^{\frac{1}{2}} C^{*} y+x\right) d \mu(y) .
$$

Proof. (1) Follows from the fact that $\mathcal{G}_{2 \theta K, I} \in G L((E))$.

(2) Since $\left\{\mathcal{G}_{2 \theta K, I}\right\}_{\theta \in \mathbb{R}}$ is differentiable, it follows that the limit

$$
\lim _{\epsilon \rightarrow 0} \frac{\mathcal{G}_{2(\theta+\epsilon) K, I} \phi-\mathcal{G}_{2 \theta K, I} \phi}{\epsilon}
$$

exists in $(E)$. Hence (2) follows.

(3) Since $\partial / \partial \theta \mathcal{G}_{2 \theta K, I} \phi=\Delta_{G}(K) \mathcal{G}_{2 \theta K, I} \phi, u(x, \theta)$ is a solution of (6.1). To show the uniqueness, let $v(x, \theta)$ be any solution of (6.1) such that (1) and $(2)$ hold. Fix $\theta \in \mathbb{R}$. Set $w(\cdot, \epsilon)=\mathcal{G}_{2(\theta-\epsilon) K, I} v(\cdot, \epsilon), \epsilon \in \mathbb{R}$. Then it easily see that $w(\cdot, \epsilon)$ is differentiable and

$$
\begin{aligned}
\frac{\partial}{\partial \epsilon} w(\cdot, \epsilon) & =-\Delta_{G}(K) \mathcal{G}_{2(\theta-\epsilon) K, I} v(\cdot, \epsilon)+\mathcal{G}_{2(\theta-\epsilon) K, I} \frac{\partial}{\partial \epsilon} v(\cdot, \epsilon) \\
& =-\mathcal{G}_{2(\theta-\epsilon) K, I} \Delta_{G}(K) v(\cdot, \epsilon)+\mathcal{G}_{2(\theta-\epsilon) K, I} \Delta_{G}(K) v(\cdot, \epsilon) \\
& =0
\end{aligned}
$$

This implies that $w(\cdot, \epsilon)=M$ (a constant) for $\epsilon \in \mathbb{R}$. Setting $\epsilon=0, \epsilon=\theta$ yields $w(\cdot, \theta)=w(\cdot, 0)=\mathcal{G}_{2 \theta K, I} v(\cdot, 0)=\mathcal{G}_{2 \theta K, I} \phi$. But $w(\cdot, \theta)=\mathcal{G}_{0, I} v(\cdot, \theta)=$ $v(\cdot, \theta)$. Hence we have $v(x, \theta)=\mathcal{G}_{2 \theta K, I} \phi(x)$ for every $x \in E^{*}$.

(4) Follows from Proposition 4.5.

Remark 6.4. A similar result of (4) in Theorem 6.3 is given in [4, Theorem 81 with different proof. 
Let $\left\{e_{k}\right\}_{k=0}^{\infty} \subset E_{\mathbb{C}}$ be a complete orthonormal basis for $H_{\mathbb{C}}$ given in Section 2 and let $\left\{a_{k}\right\}_{k=0}^{\infty} \subset \mathbb{C}$ be a sequence such that there exists $p \geq 0$, $\sum_{k=0}^{\infty}\left|a_{k} \lambda_{k}^{-p}\right|^{2}<\infty$. Then for any $\eta \in E_{\mathbb{C}}, \sum_{k=0}^{\infty} a_{k}\left\langle\eta, e_{k}\right\rangle e_{k}$ converges in $E_{\mathbb{C}}^{*}$. Moreover, the operator $K$ defined on $E_{\mathbb{C}}$ by

$$
K(\eta)=\sum_{k=0}^{\infty} a_{k}\left\langle\eta, e_{k}\right\rangle e_{k}, \quad \eta \in E_{\mathbb{C}} .
$$

is a continuous linear operator from $E_{\mathbb{C}}$ into $E_{\mathbb{C}}^{*}$.

Corollary 6.5. Let $K \in L\left(E_{\mathbb{C}}, E_{\mathbb{C}}^{*}\right)$ be defined by (6.3). Then $\left\{\mathcal{G}_{2 \theta K, I}\right\}_{\theta \in \mathbb{R}}$ is a differentiable one-parameter subgroup of $G L((E))$ whose infinitesimal generator is $\Delta_{G}(K)=\sum_{k=0}^{\infty} a_{k} D_{e_{k}}^{2}$. Moreover, $\left\{\mathcal{G}_{2 \theta K, I}\right\}_{\theta \in \mathbb{R}}$ is regular.

Corollary 6.6. Let $K \in L\left(E_{\mathbb{C}}, E_{\mathbb{C}}^{*}\right)$ be defined by (6.3) and $\phi \in(E)$. Put $u(x, \theta)=\mathcal{G}_{2 \theta K, I} \phi(x)$. Then we have

(1) $u(\cdot, \theta) \in(E)$ for any $\theta \in \mathbb{R}$.

(2) $u(\cdot, \theta)$ is differentiable with respect to $\theta$ in the topology $(E)$.

(3) $u(x, \theta)$ is a unique solution of the following equation

$$
\frac{\partial u(x, \theta)}{\partial \theta}=\left(\sum_{k=0}^{\infty} a_{k} D_{e_{k}}^{2}\right) u(x, \theta), \quad u(x, 0)=\phi(x)
$$

such that $u(x, \theta)$ satisfy (1) and (2).

(4) $u(x, \theta)$ is represented by an integral:

$$
u(x, \theta)=\int_{E^{*}} \phi\left(\sum_{k=0}^{\infty}\left(2 a_{k} \theta\right)^{\frac{1}{2}}\left\langle y, e_{k}\right\rangle e_{k}+x\right) d \mu(y) .
$$

Now we consider the following Cauchy problem:

$$
\frac{\partial u(x, \theta)}{\partial \theta}=\Delta_{G}^{*}(K) u(x, \theta), \quad u(x, 0)=\Phi(x),
$$

where $\Phi \in(E)^{*}$.

By duality of Theorem 6.2 and Theorem 6.3 , we have the following theorems. 
TheOREm 6.7. Let $K \in L\left(E_{\mathbb{C}}, E_{\mathbb{C}}^{*}\right)$. Then $\left\{\mathcal{F}_{2 \theta K, I}\right\}_{\theta \in \mathbb{R}}$ is a differentiable one-parameter subgroup of $G L\left((E)^{*}\right)$ with infinitesimal generator $\Delta_{G}^{*}(K)$.

THEOREM 6.8. Let $K \in L\left(E_{\mathbb{C}}, E_{\mathbb{C}}^{*}\right)$. Let $\Phi \in(E)^{*}$ with $\Phi(x)=\sum_{n=0}^{\infty}$ $\left\langle: x^{\otimes n}:, F_{n}\right\rangle$. Put $u(x, \theta)=\mathcal{F}_{2 \theta K, I} \Phi(x)$. Then $u(x, \theta)$ is a solution of $(6.4)$ and $u(x, \theta)$ is given by

$$
u(x, \theta)=\sum_{n=0}^{\infty}\left\langle: x^{\otimes n}: \sum_{m=0}^{[n / 2]} \frac{\theta^{m}}{m !} F_{n-2 m} \widehat{\otimes} \tau(K)^{\otimes m}\right\rangle .
$$

\section{REFERENCES}

[1] D. M. Chung and U. C. Ji, Transformation groups on white noise functionals and therr applications, to appear, Appl. Math. Opt..

[2] D. M. Chung and U. C. Ji, Some Cauchy problems in white noise analysis and associated semigroups of operators, to appear, Stoch. Anal. Appl..

[3] D. M. Chung, U. C. Ji and N. Obata, Transformations on white noise functions associated with second order differential operators of diagonal type, to appear, Nagoya Math. J..

[4] I. Dôku, H.-H. Kuo and Y. J. Lee, Fourier transform and heat equation in white noise analysis, Stochastic analysis on infinite dimensional space (H. Kunita and H.-H. Kuo, eds.), Pitman Research Notes in Mathematics Series 310, Logman Scientific \& Technical, pp. 60-74.

[5] L. Gross, Potential theory on Hilbert space, J. Funct. Anal., 1, 123-181.

[6] T. Hida, N. Obata and K. Saitô, Infinite dimensional rotations and Laplacians in terms of white novse calculus, Nagoya Math. J., 128, 65-93.

[7] T. Hida, H.-H. Kuo and N. Obata, Transformations for white noise functionals, J. Funct. Anal., 111, 259-277.

[8] T. Hida, H.-H. Kuo, J. Potthoff and L. Streit(eds.), White noise: An Infinite Dimensional Calculus, Kluwer Academic Publishers.

[9] I. Kubo and Y. Yokoi, A remark on the space of testing random variables in the white noise calculus, Nagoya Math. J., 115, 139-149.

[10] H.-H. Kuo, Gaussian Measures in Banach Space, Lect. Notes in Math., 463, Springer-Verlag, Berlin/Heidelberg/New York.

[11] H.-H. Kuo, On Laplacian operators of generalized Brownian functionals, Stochastic Processes and Applications (K. Itô and T. Hida, eds.), Lect. Notes in Math., 1203, Springer-Verlag, pp. 119-128.

[12] H.-H. Kuo, Fourier transform in white noise calculus, J. Multivar. Anal., 31, $311-327$. 
[13] H.-H. Kuo, Fourier-Mehler transform in white noıse analysis, Gaussian Random Fields (K. Ito and T. Hida, eds.), the third Nagoya Lévy Seminar, World Scientific, Singapore/New Jersey, pp. 257-271.

[14] H.-H. Kuo, Lectures on white noise analysıs, Soochow J. Math., 18, 229-300.

[15] Y.-J. Lee, Applications of the Fourier-Wiener transform to differential equations on infinite dimensional space I, Trans. Amer. Math. Soc., 262, 259-283.

[16] Y.-J. Lee, Integral transforms of analytic functions on abstract Wiener spaces, J. Funct. Anal., 47, 153-164.

[17] N. Obata, An analytic characterization of symbols of operators on white noise functıonals, J. Math. Soc. Japan, 45, 421-445.

[18] N. Obata, White noise calculus and Fock space, Lecture Notes in Math, 1577, Springer-Verlag, Berlin/Heidelberg/New York.

[19] M.A. Piech, A fundamental solution of the Parabolıc equation on Hilbert space, J. Funct. Anal., 3, 85-114.

[20] J. Potthoff and L. Streit, A characterizatıon of Hida distributions, J. Funct. Anal., 101, 212-229.

[21] J. Potthoff and J.-A. Yan, Some results about test and generalized functionals of white noise, Proc. Singapore Probab. Conf. 1989 (L. Y. Chen, ed.), Walter de Gruyter, Berlin/New York, pp. 121-145.

Dong Myung Chung

Department of Mathematics

Sogang University

Seoul, 121-742

Korea

dmchung@ccs . sogang . ac.kr

Un Cig Ji

Department of Mathematics

Sogang University

Seoul, 121-742

Korea 